\title{
Modeling of the propagation of heat in thermoelectric materials
}

\section{Nikolaev Andrey Borisovich}

Russian Federation, Honoris Causa, Doctor of Technical Sciences, Professor, Dean of the Faculty «Control Systems».

Moscow Automobile \& Road construction State Technical University, 125319, Russian Federation, Moscow, Leningradsky prospekt, 64. Tel.: +7 (499) 151-64-12. http://www.madi.ru

\section{nikolaev.madi@mail.ru}

\section{Ni Zaw}

Republic of the Union of Myanmar, Postgraduate Student, Department of «Automated Control Systems».

Moscow Automobile \& Road construction State Technical University, 125319, Russian Federation, Moscow, Leningradsky prospekt, 64. Tel.: +7 (499) 151-64-12. http://www.madi.ru

nizaw.miet@gmail.com

\begin{abstract}
This article deals with the problem of computer simulation of a nonlinear heat conduction problem, which has applications in integrated micro circuitry. We investigate the method of application of finite difference methods for solving the problem of propagation of heat in a medium with nonlinear sources (sinks) and the non-linear coefficient of thermal conductivity. Original method of averaging in the formalization of a stable finite-difference scheme, with non-linear coefficients.
\end{abstract}

Keywords: thermal conductivity, semiconductor thermoelectric material, the grid method, approximation, stability, convergence, boundary value problem. 
ISSN 2306-1561

Автоматизация и управление в технических системах (АУТС) 2014. - №1.1(8). - C. 3-13.

DOI: $10.12731 / 2306-1561-2014-1-1$

\section{УДК 681.518}

\section{Моделирование процессов распространения тепла в термоэлектрических материалах}

\section{Николаев Андрей Борисович}

Российская Федерация, Лауреат премии правительства РФ, Заслуженный деятель науки РФ, доктор технических наук, профессор, декан факультета «Управление».

ФГБОУ ВПО «Московский автомобильно-дорожный государственный технический университет (МАДИ)», 125319, Российская Федерация, г. Москва, Ленинградский проспект, д.64, Тел.: +7 (499) 151-64-12, http://www.madi.ru

nikolaev.madi@mail.ru

\section{Ни Зо}

Республика Союза Мьянма, аспирант кафедры «Автоматизированные системы управления».

ФГБОУ ВПО «Московский автомобильно-дорожный государственный технический университет (МАДИ)», 125319, Российская Федерация, г. Москва, Ленинградский проспект, д.64, Тел.: +7 (499) 151-64-12, http://www.madi.ru

nizaw.miet@gmail.com

Аннотация. Настоящая статья посвящена задаче компьютерного моделирования одной нелинейной задачи теплопроводности, имеющей приложения в интегральной микросхемотехнике. Исследуется методика применения конечно-разностных методов для решения задачи распространения тепла в среде с нелинейными источниками (стоками) и нелинейным коэффициентом теплопроводности. Описан оригинальный метод усреднения при формализации устойчивой конечно-разностной схемы с нелинейными коэффициентами.

Ключевые слова: теплопроводность, полупроводник, термоэлектрический материал, метод сеток, аппроксимация, устойчивость, сходимость, краевая задача.

\section{1. Введение}

Термоэлектрические материалы с точки зрения их технологии занимают промежуточное положение между высокоомными полупроводниками, применяемыми в радиоэлектронике (германий, кремний), и металлическими сплавами. Возобновление широкого интереса к проблеме создания эффективных термоэлектрических 
преобразователей связано с внедрением новых подходов, в первую очередь, основанных на концепции Слэка о веществах, сочетающих свойства фононного стекла и электронного кристалла в результате пространственного разделения подструктур, отвечающих за транспорт носителей заряда и транспорт фононов [1 - 4]. В результате оказывается возможным практически независимое увеличение электропроводности и уменьшение фононной теплопроводности материала, что необходимо для реализации высоких значений безразмерной термоэлектрической эффективности.

\section{2. Теплопроводность термоэлектрических материалов}

Теплопроводность представляет собой одно из важнейших физических свойств полупроводникового металла, характеризующее способность тела передавать тепловую энергию от одной его точки к другой, если между ними существует разница температур. Электрические проводники и диэлектрики имеют различные механизмы теплопроводности, и, как следствие, температурные зависимости теплопроводности в них носят различный характер. Знание теплопроводности полупроводникового металла необходимо для проведения теплофизических расчетов [1 - 13].

Рассмотрим процесс распространения тепла в однородном стержне. На рисунке 1, представлена физическая модель, представляющая собой тонкий металлический стержень (брус), нагреваемый с одного или с обоих торцов [1].

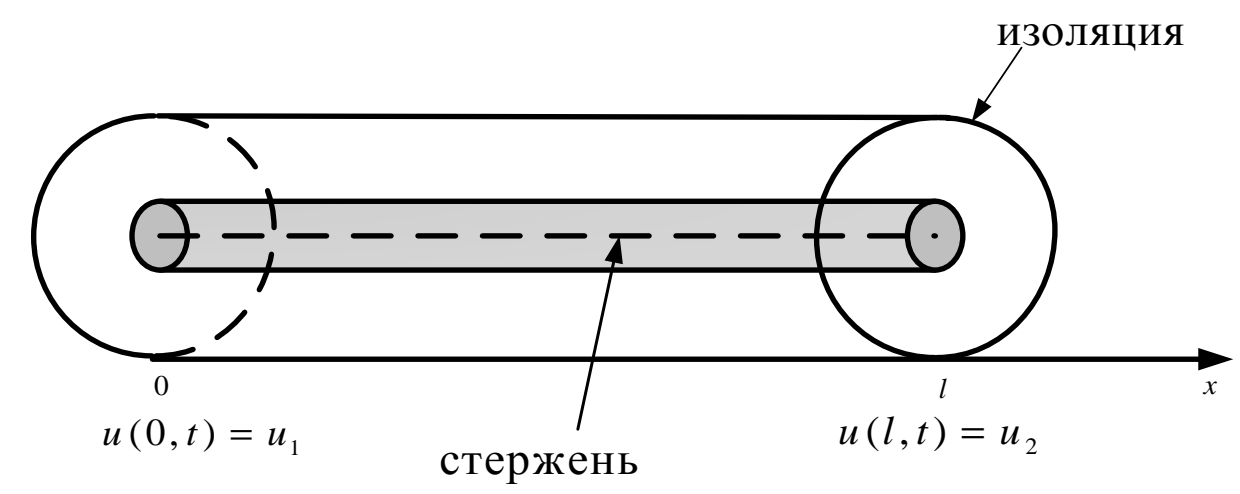

\section{Рисунок 1 - Физическая модель распространения тепла в однородном стержне}

При условии боковой изоляции стержня потери тепла через боковую поверхность отсутствуют. Поэтому, если считать, что теплоемкость стержня постоянна (однородный стержень), то температура в любом поперечном сечении будет зависеть лишь от координаты х и от времени $t$. Этот процесс может быть описан функцией $u(x, t)$, представляющей температуру в сечении $x$ в момент времени $t$. Найдем уравнение, которому должна удовлетворять функция $u(x, t)$. Для этого сформулируем физические закономерности, определяющие процессы, связанные с распространением тепла [1 - 4].

Изучение температурных процессов в большом интервале изменения температур приводит к нелинейному уравнению теплопроводности в однородном стержне (однородной одномерной среде) запишется в виде: 


$$
c \rho \frac{\partial u}{\partial t}=\frac{\partial}{\partial x}\left(k(u, x) \frac{\partial u}{\partial x}\right)+F(u, x, t)
$$

где $c$ - удельная теплоемкость, $\rho$ - плотность, $k(u, x)$ - коэффициент теплопроводности (в общем случае зависит от самого решения $u(x, t)$ ). Тепловой поток через боковую поверхность отсутствует. Внутри среды действуют источники или стоки, плотность которых описывается функцией $F(u, x, t)$.

Для выделения единственного решения уравнения теплопроводности необходимо к уравнению присоединить начальные и граничные условия. Начальное условие в отличие от уравнения гиперболического типа состоит лишь в задании значений функции $u(x, t)$ в начальный момент $t_{0}$. Граничные условия могут быть различны в зависимости от температурного режима на границах. Рассматривают три основных типа граничных условий.

$$
\begin{gathered}
u(x, 0)=\varphi(x) \text { - начальное условие; } \\
\left\{\begin{array}{l}
\left.\alpha_{1} \frac{\partial u}{\partial x}\right|_{x=0}+\beta_{1} u(0, t)=q_{1}(t) \\
\left.\alpha_{2} \frac{\partial u}{\partial x}\right|_{x=1}+\beta_{2} u(1, t)=q_{2}(t) .
\end{array}\right.
\end{gathered}
$$

При $\alpha_{1}=0, \beta_{1}=1$ - граничное условие первого рода. При $\alpha_{1}=1, \beta_{1}=0$ граничное условие второго рода. Если $\alpha_{1} \neq 0, \beta_{1} \neq 0$ - граничное условие третьего рода, где - $\beta$ - параметр определяющей степенную зависимость функции поглощения от температуры; $\alpha$ - коэффициент температур-опроводности

Теоретической основой для численного решения поставленной задачи является метод сеток, в котором основными понятиями являются аппроксимация, устойчивость и сходимость конечно-разностных уравнений к точному решению исходной дифференциальной задачи при стремлении шагов сетки $h$ к нулю. Изобразим сетку (рисунок 2), на которой мы ищем решение дифференциальной задачи:

$$
L u=f .
$$

Сеточная система уравнений:

$$
L_{h} u^{(h)}=f^{(h)},
$$

где $L_{h}$ - конечно-разностная аппроксимация дифференциального оператора; $u^{(h)}$ значения функции $u(x, t)$ в узлах сетки (матрица).

\section{3. Определение сходимости}

Пусть требуется приближенно вычислить решение и дифференциальной краевой задачи (3)

Для приближенного отыскания таблицы $[u]_{h}$, представляющей собой точное решение задачи (3), надо на основе этой задачи составить систему уравнений вида (4) относительно функции $u^{(h)}$ из $U_{h}$, чтобы имела место сходимость 
$\mid[u]_{h}-u^{(h)} \|_{U_{h}} \rightarrow 0, h \rightarrow 0$. Если для решения разностной краевой задачи (4) выполнено неравенство $\mid[u]_{h}-u^{(h)} \|_{U_{h}} \leq c h^{k}, c=$ const . Сходимость имеет порядок $k$ относительно $h$.

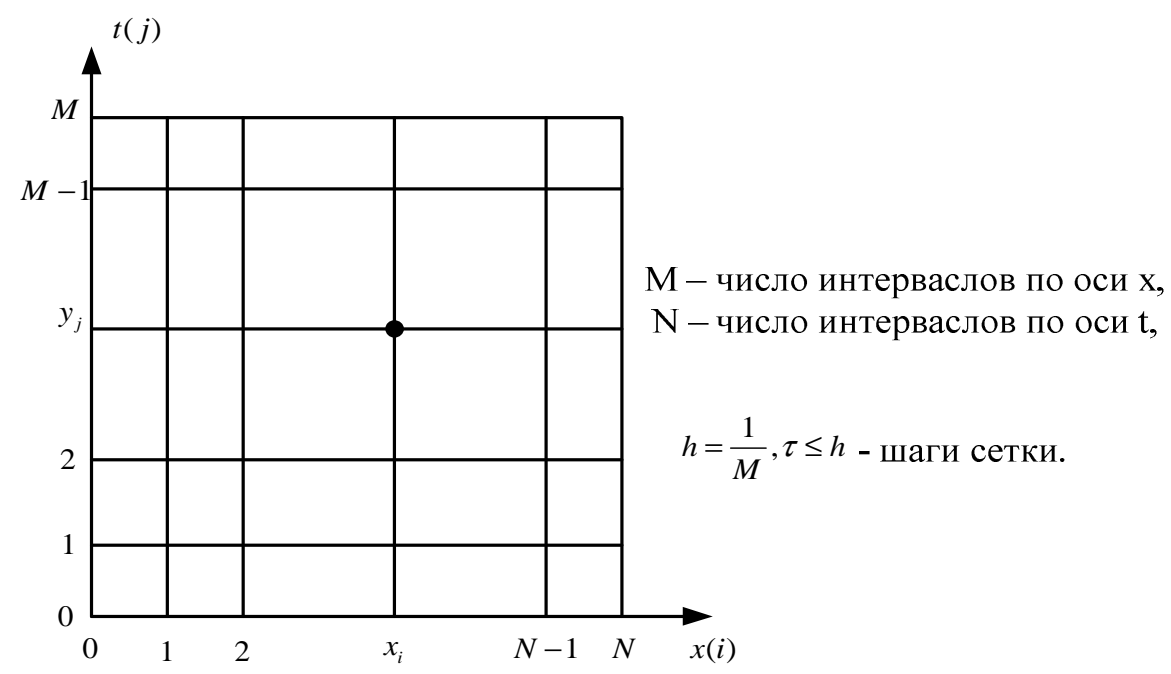

\section{Рисунок 2 - Сетка для аппроксимации дифференциальной задачи}

\section{4. Аппроксимация}

Чтобы понятие аппроксимации имело смысл, надо ввести норму в пространстве $F_{h}$, которому принадлежит правая часть $f^{(h)}$ уравнения (4). По определению разностная задача (4) аппроксимирует задачу (3) на решении u, если в равенстве $L_{h}[u]_{h}=f^{(h)}+\delta f^{(h)}$, невязка $\delta f^{(h)}$, возникающая при подстановке $[u]_{h}$ в разностную краевую задачу (4), стремится к нулю, при $h \rightarrow 0:\left\|\delta f^{(h)}\right\|_{F_{h}} \rightarrow 0 \quad\left\|\delta f^{(h)}\right\|_{F_{h}} \leq c h^{k}$. Где с не зависит от $\mathrm{h}$, то аппроксимация имеет порядок $\mathrm{k}$ относительно $\mathrm{h}$.

Определение устойчивости

Разностная краевая задача (4) по определению устойчива, если существуют числа $\delta>0, h_{0}>0$ такие, что при любом $h<h_{0}$ и любом $\varepsilon^{(h)}$ из $F_{h}$, удовлетворяющем неравенству $\left\|\varepsilon^{(h)}\right\|_{F_{h}}<\delta$, разностная краевая задача $L_{h} z^{(h)}=f^{(h)}+\varepsilon^{(h)}$, имеет одно и только одно решение, причем выполняется условие $\left\|z^{(h)}-u^{(h)}\right\|_{U_{h}} \leq c\left\|\mathcal{E}^{(h)}\right\|_{F_{h}}$. где $c$ некоторая постоянная, не зависящая от $h$.

На рисунке 3 показаны варианты выбора шаблонов на сетке, обычно используемых при решении линейных задач теплопроводности. Здесь же приведена конечно-разностная система уравнений, аппроксимирующая дифференциальную задачу с постоянными коэффициентами на шаблоне “ящик” и имеющая повышенный порядок точности $O\left(\tau_{2}+h_{2}\right)$. 


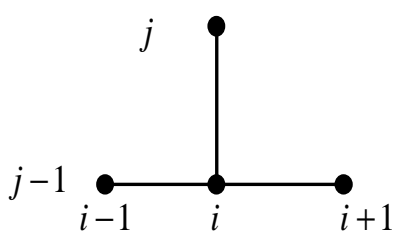

a)

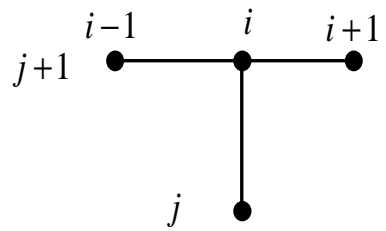

б)

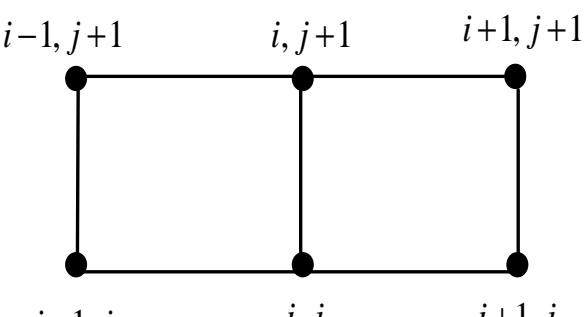

B)

Рисунок 3 - Конечно-разностные схемы и виды шаблонов: а) образный шаблон для явной схемы; б) образный шаблон для неявной схемы; в) схема «КранкаНиколсона» (схема «ящик»)

Схемы а) и б) дают точность аппроксимации $O\left(\tau_{2}+h_{2}\right)$. Схема в) при значении весовых коэффициентов $=\frac{1}{2}=1-\sigma$ дает точность $O\left(\tau_{2}+h_{2}\right)$. Соответствующая конечно-разностная система для этой схемы имеет вид:

$$
\frac{u_{i, j+1}-u_{i, j}}{\tau}=\frac{u_{i-1, j+1}+u_{i+1, j+1}}{2 h^{2}}+\frac{u_{i-1, j}-2 u_{i, j}+u_{i+1, j}}{2 h^{2}}+f_{i, j+1 / 2},
$$

где

$$
f_{i, j+1 / 2}=f(i h,(j+1 / 2) \tau) .
$$

Эта схема является неявной. Для решения поставленной нелинейной задачи предлагается использовать модификацию интегро-интерполяционного метода дискретизации дифференциальной задачи. Метод заключается в интегрировании основного уравнения по элементарной ячейке сетки $\prod_{i, j}$ и использовании тех или иных квадратурных формул интерполяционного типа. Результирующая конечно-разностная система уравнений имеет порядок точности $O\left(\tau+h_{2}\right)$.

Рассмотрим приведенное уравнение нелинейной теплопроводности общего вида:

$$
\frac{\partial u}{\partial t}=\frac{\partial}{\partial x}\left(k(u) \frac{\partial u}{\partial x}\right)+f(u)
$$

Проинтегрируем обе части данного уравнения по элементарной ячейке сетки, т.е. по малому прямоугольнику $\Pi_{i, j}$ имеет вид:

$$
\iint_{\Pi_{i j}} \frac{\partial u}{\partial t} d x d t=\iint_{\Pi_{i j}} \frac{\partial}{\partial x}\left(k(u) \frac{\partial u}{\partial x}\right) d x d t+\iint_{\Pi_{i j}} f(u) d x d t
$$

Распишем подробно интеграл в левой части:

$$
\begin{aligned}
& \iint_{\Pi_{i j}} \frac{\partial u}{\partial t} d x d t=\int_{t_{j}}^{t_{j+1}} d t \int_{x_{i}}^{x_{i+1}} \frac{\partial u}{\partial t} d x=\int_{t_{j}}^{t_{j+1}} d t \frac{\partial}{\partial t} \int_{x_{i}}^{x_{i+1}} u d x=\int_{t_{j}}^{t_{j+1}} \frac{\partial}{\partial t}\left(u\left(x_{i}, t\right)\right) h+\tau o\left(h^{2}\right)=\left.h u\left(x_{i}, t\right)\right|_{t_{j}} ^{t_{j+1}}+\tau o\left(h^{2}\right)= \\
& =h\left(u\left(x_{i}, t_{j+1}\right)-u\left(x_{i}, t_{j}\right)\right)+\tau o\left(h^{2}\right)=h\left(u_{i, j+1}-u_{i, j}\right)+\tau o\left(h^{2}\right)
\end{aligned}
$$


При вычислении интеграла от правой части, т.е. от функции $f(u)$, используем формулу трапеций при интегрировании по $x$ и формулу прямоугольника при интегрировании по $t$. В результате получаем:

$$
\iint_{\Pi_{i j}} f(u) d x d t=\int_{t_{j}}^{t_{j+1}} d t \int_{x_{i}}^{x_{i+1}} f(u) d x=\tau h \hat{f}_{i, j}+\tau o\left(h^{3}\right)
$$

где

$$
\begin{gathered}
\hat{f}_{i, j}=\frac{1}{2}\left(f\left(u_{i, j}\right)+f\left(u_{i+1, j}\right) .\right. \\
\int_{t_{j}}^{t_{j+1}} d t \int_{x_{i}}^{x_{i+1}} \frac{\partial}{\partial x}\left(k(u) \frac{\partial u}{\partial x}\right) d x=\frac{\tau}{h} k_{1}\left(u_{i+1, j+1}-2 u_{i, j+1}+u_{i-1, j+1}\right)+2 \frac{\tau}{h} k_{2}\left(u_{i+1, j+1}-u_{i, j+1}\right)+\tau o\left(h^{2}\right)+o\left(\tau^{2}\right) \\
\text { где } \\
\hat{f}_{i, j}=\frac{1}{2}\left(f\left(u_{i, j}\right)+f\left(u_{i+1, j}\right), k_{1}=\frac{k_{i, j}+k_{i+1, j}}{2}, k_{2}=\frac{k_{i+1, j}-k_{i, j}}{2}, k_{i, j}=k\left(u_{i, j}\right),\right.
\end{gathered}
$$

a устойчивость полученной схемы исследуется методом “замороженных коэффициентов”.

Отбрасывая бесконечно малые и, заменяя точное сеточное решение $u_{m, j}$ на приближенное $y_{m, j}$, получим окончательно следующую неявную конечно-разностную схему:

$$
y_{m, j+1}-y_{m, j}=\mathcal{Y} k_{1}\left(y_{m+1, j+1}-2 y_{m, j+1}+y_{m-1, j+1}\right)+2 k_{2}\left(y_{m+1, j+1}-y_{i, j+1}\right)+\tau \bar{f}_{m, j} .
$$

Для исследования устойчивости по правой части ищутся частные решения в виде гармоник произвольной частоты $y_{m, j}=\lambda_{j} e_{i} m \varphi$. При исследовании модуля $\lambda$ как функции от $\varphi, k_{1}, k_{2}$ коэффициенты $k_{1}$ и $k_{2}$ “размораживаются” и показывается, что $|\lambda|<1$ при любых $k_{1}$ и $k_{2}$.

Далее полученная конечно-разностная трехдиагональная система приводится к стандартному виду, показанному в выражении (11), и решается методом прогонки по временным слоям. Рассмотрим первую краевую задачу для приведенного нелинейного уравнения со степенными зависимостями основных характеристик:

$$
\left\{\begin{array}{l}
\frac{\partial u}{\partial t}=\frac{\partial}{\partial x}\left(k(u) \frac{\partial u}{\partial x}\right)+q f(u) \\
u(x, 0)=\phi(x), \\
u(0, t)=u_{0}, \\
u(1, t)=u_{1}, \\
x \in[0,1]
\end{array} .\right.
$$

Рассмотрим следующий случай физически реализуемой степенной зависимости коэффициента температуропроводности от температуры: 


$$
\begin{gathered}
\sim 10 \sim \\
k(u)= \begin{cases}1+u^{\sigma}, & u \geq 0, \\
1, & u<0 ; \quad \sigma \geq 1 .\end{cases}
\end{gathered}
$$

Для функции источника (стока) также примем степенную зависимость от температуры: $f(u)=q u^{\beta} ; \beta \geq 1$, где $q=1$ соответствует источнику, а $q=-1$ - стоку. Наличие источников подобного типа чаще всего определяется разогревом проводящего материала при прохождении токов высокой частоты, а наличие стоков эндотермическими (химическими) реакциями в материале, идущими с поглощением тепла.

Учитывая полученную выше аппроксимацию уравнения (11) на двумерной сетке $\omega_{h, \tau}=\left\{\left(x_{i}, t_{j}\right) \mid x_{i}=i h, \quad i=0,1, \ldots, M ; \quad h=\frac{1}{M} ; t_{j}=j \tau, j=0,1, \ldots, N ; \tau=1 / N, \quad \tau \leq h\right\}_{\text {в }}$ форме неявной схемы (10), получаем конечно-разностную систему с трехдиагональной матрицей. Преобразуем (10) к стандартному виду:

$$
\begin{gathered}
\left\{\begin{array}{l}
-a_{i, j} y_{i-1, j+1}+c_{i, j} y_{i, j+1}-b_{i, j} y_{i+1, j+1}=\bar{f}_{i, j}, \\
i=1,2, \ldots, M-1 ; \\
y_{0, j}=u_{0}, j=0,1, \ldots, N ; \\
y_{i, N}=u_{1}, i=0,1, \ldots, M ; \\
y_{i, 0}=\phi_{i}=\phi(i h), i=0,1, \ldots, M ;
\end{array}\right. \\
\left\{\begin{array}{l}
a_{i, j}=\gamma k_{1}, b_{i, j}=\gamma\left(k_{1}+2 k_{2}\right), \\
c_{i, j}=1+2 \gamma\left(k_{1}+k_{2}\right), \bar{f}_{i, j}=y_{i, j}+\frac{\tau}{2}\left(f\left(y_{i, j}\right)+f\left(y_{i+1, j}\right)\right) .
\end{array}\right.
\end{gathered}
$$

Для успешной реализации метода прогонки необходимо выполнение условия устойчивости:

$$
\left|a_{i, j}\right|+\left|b_{i, j}\right| \leq\left|c_{i, j}\right|
$$

\section{5. Вычислительный эксперимент и его результаты}

На рисунках 4 и 5 показаны результаты вычислительного эксперимента, реализованного в пакете MATLAB с помощью специально разработанных программ nonlinearheat и nonlinearheat1. Первая из них предназначена для решения первой краевой задачи методом прогонки, вторая - для решения краевой задачи с условием второго рода на левом конце отрезка (условием отсутствия теплообмена через левый торец стержня). Наиболее интересные результаты получаются для второго случая.

В качестве начального распределения температуры при $t=0$ были рассмотрены две функции: первая из них имеет участок отрицательных температур на половине длины стержня, вторая - имеет квадратичную зависимость начальной температуры от $O$ до $u_{1}$. 


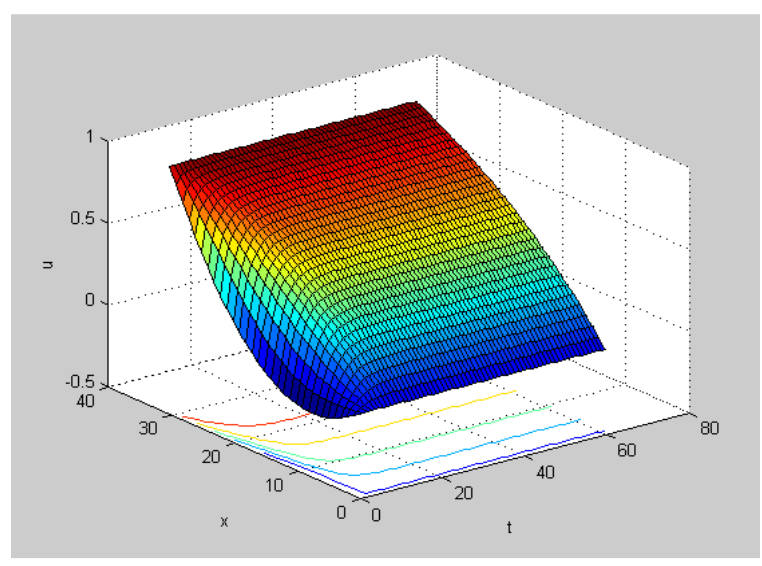

графический образ матрицы $y_{i j}$

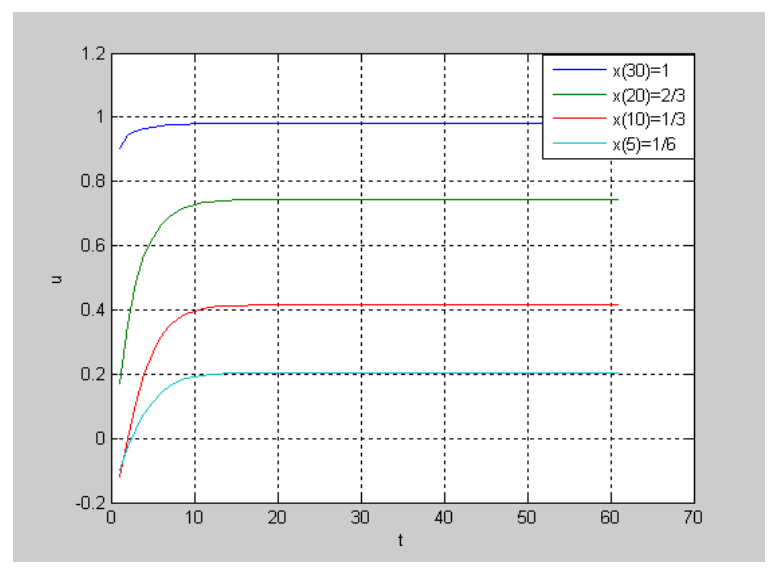

зависимости температуры от $\boldsymbol{t}$ при фиксированном $\boldsymbol{x}$

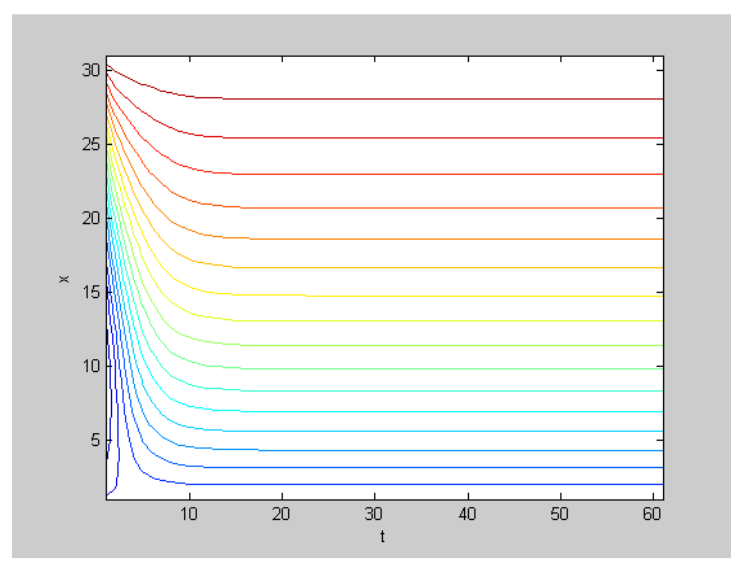

линии уровней матрицы $y_{i j}$

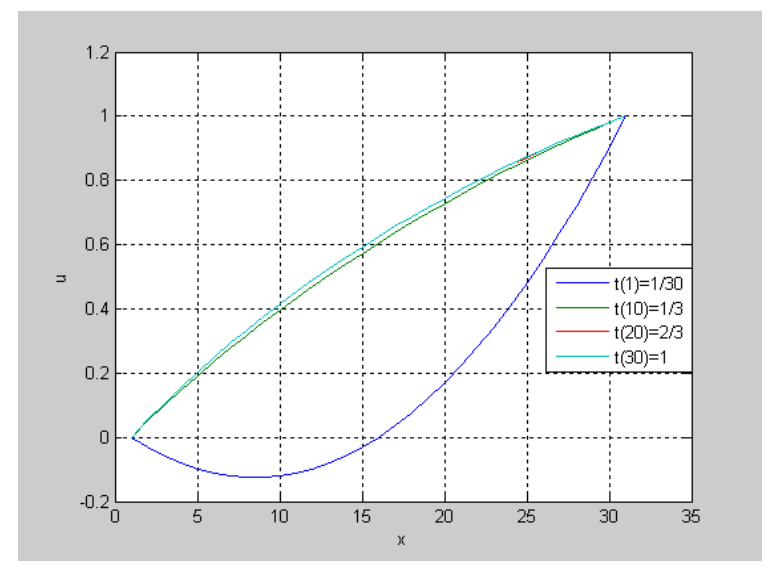

зависимости температуры от $\boldsymbol{x}$ при фиксированном $t$

Рисунок 4 - Графики срезов матрицы $Y=\left[y_{i j}\right]$ :

$$
\text { sig }=1 ; \text { beta }=1 ; l e v=2 ; \text { del }=1.0 ; m=30 ; n=90
$$

В программах предусмотрена ситуация, когда при вводе слишком больших значений $u_{l}$ (температуры на правом конце стержня) нарушается условие устойчивости метода прогонки. В этом случае для преодоления неустойчивости необходимо уменьшать шаг $\tau$ по оси времени, что автоматически приводит к уменьшению промежутка существования решения по времени.

В программе MATLAB входными параметрами являются:

$m$ - число интервалов равномерной сетки по оси $o x$;

$n$ - число интервалов равномерной сетки по оси оу (оси времени);

sig - определяет степенную зависимость коэффициента температуропроводности;

beta - определяет степенную зависимость функции источника (стока);

$d e l$ - коэффициент уравнения шага $\tau$ по оси времени;

$z n a k=1$, если включено источник и $z n a k=-1$, если включен сток;

$l e v$ - определяет температуру, заданную на правом конце стержня. 


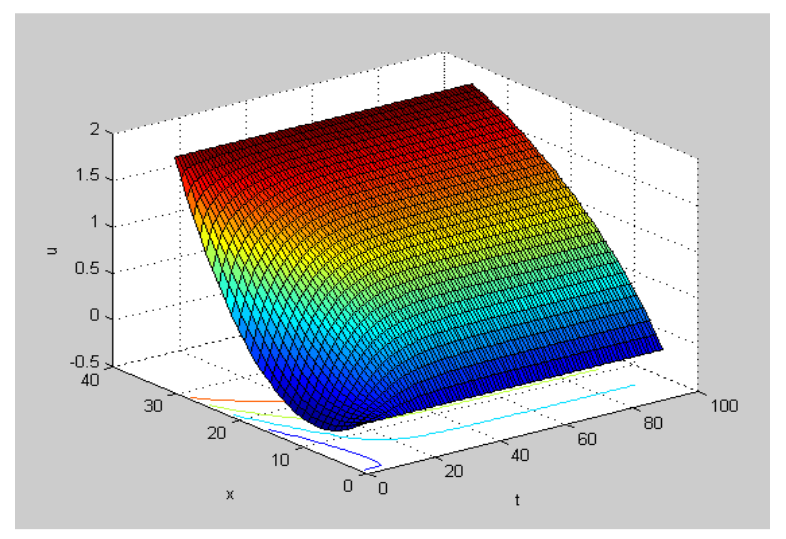

графический образ матрицы $y_{i j}$;

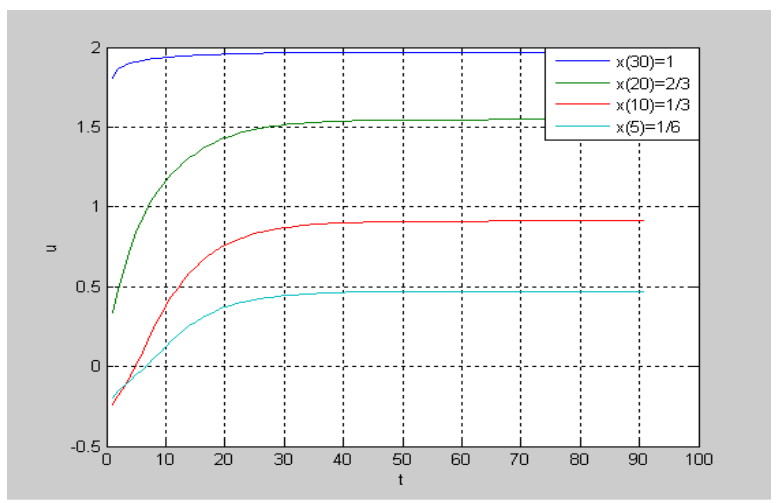

зависимости температуры от $\boldsymbol{t}$ при фиксированном $\boldsymbol{x}$

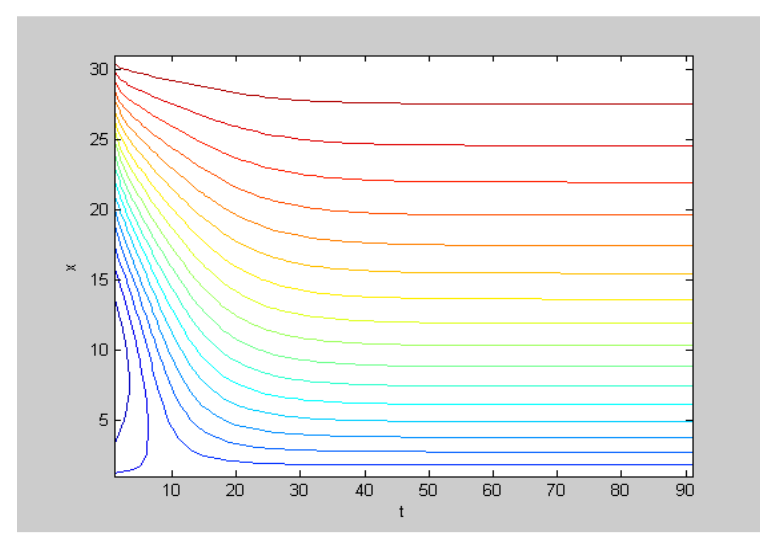

линии уровней матрицы $y_{i j}$;

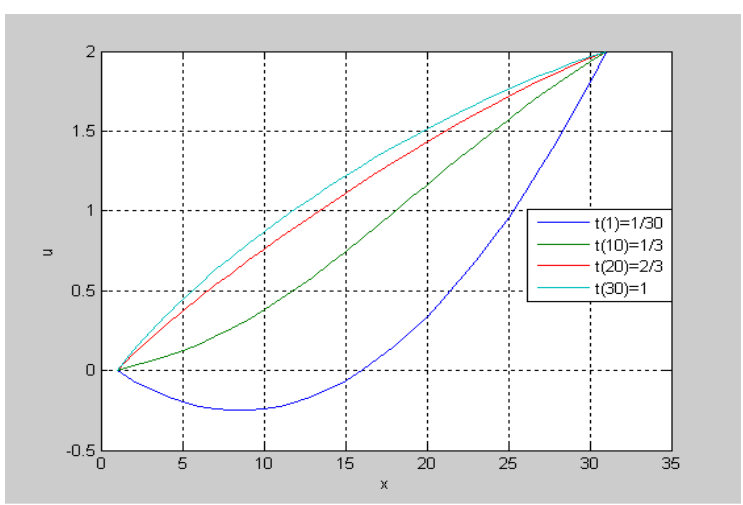

зависимости температуры от $\boldsymbol{x}$ при фиксированном $t$

$$
\begin{gathered}
\text { Рисунок } 5 \text { - Графики срезов матрицы } \mathrm{Y}=\left[y_{i j}\right]: \\
\text { sig }=1 ; \text { beta }=2 ; l e v=2 ; \text { del }=0.2 ; m=30 ; n=90
\end{gathered}
$$

\section{6. Заключение}

Анализ полученных результатов раскрывает следующие особенности поведения решения:

1) В целом, при правильном выборе отношения шагов сетки $\tau / h \leq 1$, графики демонстрируют непрерывную зависимость решения от параметров $\sigma, \beta$ и начальных данных.

2) При увеличении параметра $\beta$ в диапазоне (2 - 3) наблюдается увеличение скорости роста температуры от времени, приводящее при некоторых значениях lev > $l e v_{k p}$ к срыву прогонки вследствие появления неустойчивости. При этом, чем больше $\beta$, тем меньше соответствующее значение $l e v_{k p}$ и тем меньшее значение шага $\tau$ следует задавать для преодоления неустойчивости, если фиксируется число узлов $\mathrm{n}$ по оси времени.

Например, из рисунков 4 и 5 видно, что при $\sigma=1, \varphi(x)=\operatorname{poll}(x)$ шаг $\tau_{\text {по оси }}$ времени пришлось взять в пять раз меньшие для $\beta=2$, чем для $\beta=1$. 
Аналогичная ситуация наблюдается и при другом наборе параметров для фиксированных значений $\sigma, l e v$ и $\varphi(x)$.

\section{Список информационных источников}

[1] Пилипенко В.А. Быстрые термообработки в технологии СБИС. Мн.: Изд. центр БГУ. - 2004. - С. 24.

[2] Ни Зо. Использование нечетких регуляторов в системах управления технологическими процессами при производстве полупроводниковых микросхем // Автоматизация и управление в технических системах. - 2012. - № 2. - С. 22-28. URL: auts.esrae.ru/2-18 (дата обращения: 22.12.2013).

[3] Ни Зо, Николаев А.Б. моделирование полупроводниковых микросхем с использованием термоэлектрического способа // Автоматизация и управление в технических системах. - 2012. - № 2. - С. 28-38. URL: auts.esrae.ru/2-19 (дата обращения: 22.12.2013).

[4] Николаев А.Б., Ни Зо. Исследование системы автоматического управления термоэлектрическим объектом // Автоматизация и управление в технических системах. - 2013. - № 4.1. - C. 113-120. DOI: 10.12731/2306-1561-2013-4-18.

[5] Остроух А.В. Основы построения систем искусственного интеллекта для промышленных и строительных предприятий: монография / А.В. Остроух. - М.: OОО «Техполиграфцентр», 2008. - 280 с. - ISBN 978-5-94385-033-2.

[6] Остроух А.В. Информационные технологии в научной и производственной деятельности / [ред. А.В. Остроух] - М: ООО "Техполиграфцентр", 2011. - 240 с. ISBN 978-5-94385-056-1.

[7] Остроух А.В. Ввод и обработка цифровой информации: учебник для нач. проф. образования / А.В. Остроух. - М.: Издательский центр «Академия», 2012. - 288 с. - ISBN 978-5-7695-9457-1.

[8] Остроух А.В. Исследование начального периода моделирования на точность среднеинтегральной оценки имитационных моделей / А.В. Остроух, А.А. Солнцев, Н.В. Солдатов, К.А. Новицкий, П.С. Якунин // Вестник МАДИ. - 2010. Вып. 2(21). - С. 61-65.

[9] Остроух А.В. Системы искусственного интеллекта в промышленности, робототехнике и транспортном комплексе: монография / А.В. Остроух Красноярск: Научно-инновационный центр, 2013. - 326 с. - ISBN 978-5-90631410-9.

[10] Nickolayev A.B., Ostroukh A.V., Zamytskikh P.V., Gubanov A.I. Automated system of oil quantity and quality indexes estimation // EUROPEAN JOURNAL OF NATURAL HISTORY. - 2011. - № 3 - C. 96-98.

[11] Остроух А.В., Тянь Юань. Современные методы и подходы к построению систем управления производственно-технологической деятельностью промышленных предприятий // Автоматизация и управление в технических системах. - 2013. - № 1(3). - C. 29-31.

[12] Остроух А.В. Интеграция компонентов системы мониторинга / А.В. Остроух, Юань Тянь // Молодой ученый. - Чита: ООО «Издательство Молодой ученый», 2013. - №10. - С. 182-185.

[13] Остроух А.В., Николаев А.Б., Сальный А.Г., Кухаренко В.Н. Общие принципы построения SCADA-систем // Автоматизация и управление в технических системах. - 2013. - № 2(4). - С. 8-12. 